\title{
Training in General Surgery Ward Call: A Resident-Student Buddy System
}

\author{
Andrew Maurice, Angus Hann
}

Royal Brisbane and Women's Hospital, Australia

\begin{abstract}
There is a paucity of literature regarding medical student experiences of after hours hospital ward call. It was observed at our institution that medical students had minimal experience in ward call, yet were required to undertake such shifts as interns after graduation. We implemented a buddy system in which a medical student shadowed a general surgery resident for a ward call shift.

Final year medical students were recruited from the local university at a tertiary teaching hospital after institutional approval. Each student attended a 4 hour evening shift on a general surgery ward with a supervising resident. A survey detailing attitudes and expectations of ward call was completed before and after the experience.
\end{abstract}

Nine students enrolled in the project. Familiarity of expectations of what is required of an intern on a ward call shift improved significantly after the experience $(3.1 / 5$ to $4.1 / 5, p=0.002)$. After hours work experience was reported as useful both before and after the study $(4.5 / 5$ to $4.7 / 5, p$ $=0.47$ ). Students and doctors involved unanimously felt the experience was worthwhile.

After hours ward call experience is useful for a final year medical student. More studies are required to further define the role of after hours ward call experiences during medical training.

\section{Problem}

In Australia, all medical practitioners complete an internship following graduation. This consists of numerous hospital placements (or "terms") over a period of 12 months and includes at least one surgical, medical, and emergency medicine term. Most doctors opt to complete at least one further year as a resident medical officer after internship. The United Kingdom employs a similar system for junior doctors in their two year "Foundation" program.

The majority of work in these roles is typically comprised of assisting their unit during normal working hours. This may involve basic procedures, ward rounds, clinic and general administrative tasks in a supervised environment where decisions are made by a senior doctor and tasks delegated to the junior doctors.

Interns and resident medical officers are also required to cover work on hospital wards after hours, known as "ward call" at our institution. The medical officers responsibilities on these shifts typically include reviewing unwell or deteriorating patients, assisting at emergency calls or "codes", insertion of intravenous lines, charting medications, and adjusting intravenous fluids or infusions. In contrast to the normal "day job" tasks of internship, ward call typically involves the intern assessing patients, prescribing medications, and performing procedures without the immediate supervision of a more senior clinician.

The medical program at the University of Queensland generally assigns medical students to a medical, surgical, or other inpatient medical team in which the student is an observer of the day to day activities during normal working hours. At present, students are encouraged but not required to attend ward call shifts.

We feel that medical students gain excellent exposure to the normal activities and tasks of a hospital team during working hours, however have limited exposure to ward call and after hours workings of the hospital in general. This presents a potential danger because after hours shifts involve decision making without direct supervision. In addition, significantly reduced staffing means that senior assistance is often harder to obtain.

Furthermore, it is anecdotally well known that junior doctors typically dread their initial ward call shifts due to the potential stressful situations that may arise with limited supervision and assistance.

Thus we aimed to quantify the perceptions of medical students with respect to ward call as well as trial a "buddy system" in which the student could attend a ward call shift with an experienced resident medical officer.

\section{Background}

There is a small amount of published literature regarding after hours medical student experiences and it predominately comes from institutions in the United States.

In 2009, a study demonstrated that $61 \%$ of American medical schools were providing an after hours call experience to final year medical students (REF 1). A 2014 survey of 28 final year medical students found that only a minority had undertaken any night call experience and the majority found the experience useful (REF 2). Another survey of 167 students in 2013 found that $59 \%$ of students 
who responded to the survey had experience in night call work and were more likely to feel it was worthwhile in comparison to those that did not experience night call (REF 3).

There is no published data regarding which medical programs within Australia require students to attend after hours shifts and in what capacity. To the best of our knowledge, there is no published data examining the experience of medical students in after hours shifts, in particular ward call, in the Australian setting.

\section{Baseline measurement}

For students who had agreed to participate in the program, a survey was performed. The survey consisted of questions regarding the students level of anxiety regarding future ward call shifts as well as what their level of familiarity and expectations were of ward call work. The same survey was completed after the ward call experience.

An example is included as Figure 1.

See supplementary file: ds5931.docx - "Figures 1, 2 and 3"

\section{Design}

This quality improvement study gave senior medical students a chance to 'buddy up' with a senior resident and experience a shift of ward call.

With university permission, a list of students undertaking a surgical rotation at the Royal Brisbane and Women's Hospital between June and August 2014 was obtained. All students were in their fourth year of a four year Bachelor of Medicine / Bachelor of Surgery degree at the University of Queensland, Brisbane, Australia.

Of the 45 students emailed, ten volunteered to be part of the study. One of the ten students was unable to attend their session, thus a total of nine students attended a session.

Each student was partnered with a resident (one of the authors of this study) for an evening shift of general surgery ward call from $6 \mathrm{pm}$ until $10 \mathrm{pm}$. This involved covering a sixty bed general surgery ward and any general surgery outliers in the hospital.

As a senior medical student, the students were given the opportunity to perform intravenous cannulation and venepuncture, chart medications, intravenous fluids, heparin, insulin as well as complete radiology and pathology requests. All orders were approved and co-signed by the supervising resident. The students were also given the opportunity to review, examine, and formulate management plans for patients. The resident supervised all activities and each student undertook one shift only.

The general surgery department was chosen as the preferred unit for this study for several reasons. Firstly, all interns are required to undertake a term in surgery and this is most likely to be general surgery. Secondly, all interns are likely to be faced with general surgery patients on ward call. Thirdly, the general surgery unit has a wide variety of patient pathology in comparison to a sub-specialty unit. The final and most important reason the general surgery unit was chosen was because it has a high volume of 'common ward call tasks' such as prescribing fluids, heparin, insulin, and patient reviews (for example post-operative fever and oliguria) in comparison to other wards in the hospital. It was thus felt the general surgery ward would provide the optimal experience for a student wishing to experience a ward call shift.

\section{Strategy}

After the first three students, it was clear that it was difficult to keep track of what activities the students were exposed to in their shift. Thus we devised a 'check-list' of common ward call activities that we felt were important for students to be exposed to. This included simple tasks such as intravenous cannulation as well as more difficult tasks such as assessment of patients or assisting at a Medical Emergency. An example of a completed check-list is included as Figure 2

Around the middle of this project, it was clear that most students did not know what was expected on a ward call shift. This meant that for the first 30 minutes of the ward call shift, the resident would have to explain to the student exactly what was required on a ward call shift and what the expected responsibilities of the medical officer are. This was difficult to do if a night was particularly busy. Thus we created a two-page summary entitled 'What to Expect on Ward Call' which we gave to the student after they had completed the initial survey but before their shift. This allowed us to spend less time in orientating the medical student.

See supplementary file: - "PDSA Cycles"

See supplementary file: ds5906.docx - "PDSA Cycles"

\section{Post-measurement}

As described, a survey was compared to before and after the survey. We also allowed the students to write free text comments. A ward-call check-list was also used as described above (Figure 2).

A table with the overall results is presented in Table 1. Welch's paired t-test assuming unequal variances was used to determine if there was a statistically significant difference between the pre- and post-survey results. The $p$-values are given in Table 1 .

The overall study results are presented for PDSA cycles 1-3. Combined changes after PDSA cycle 2-3 are also shown in the table (recall PDSA cycle 2 and 3 involved the use of a sign off sheet as well and orientation materials to better prepare the student).

Overall, the students improved their knowledge of what to expect during ward call shifts $(3.1 / 5$ to $4.1 / 5, p=0.002)$. The students felt the experience was worthwhile before and after the experience $(4.5 / 5$ to $4.7 / 5, p=0.472)$. Confidence in prescribing, emergency calls and knowing how to call for help improved after the 
experience. When asked if medical school had prepared the student for ward call, the response did not change (2.8/5 to $2.7 / 5$, $\mathrm{p}=0.824$ ).

When examining combined PDSA cycles 2 and 3 , it can be observed that the trends are essentially unchanged for these cycles, however a few of the questions lose statistical significance, likely due to sample size reducing from nine to six students. PDSA cycle 2 and 3 results have not been presented on their own as trends are virtually identical but most results are not statistically significant given the small sample size.

Written comments were uniformly positive and most centered on a perceived lack of training in the after hours environment. A selection of comments has been included (Figure 3 ).

The subjective experience of the residents who agreed to buddy with students (i.e. the authors of this study) was positive. Having a student can lighten the workload on an after hours shift. For instance, a student can assist with filling out pathology requests that can then be checked and signed by the resident. It was also great to work with motivated medical students who volunteered their free time. It was also interesting to see that students had very little understanding or knowledge of what a ward call shift was and what was expected.

It was observed that several students did not have a chance to see a Medical Emergency (i.e. Code Blue or 'Medical Emergency Response Team' call) during their term. Furthermore, the experience for the student was sub-optimal if the shift was either extremely busy or quiet. On a busy shift, it is difficult to have time to explain to the student what the resident was doing or to delegate a critical task (e.g. urgent venepuncture). Thus, we propose that at least 2 or more shifts should be undertaken to ensure the student has an adequate experience.

See supplementary file: ds5976.docx - "Table 1: results"

\section{Lessons and limitations}

We discovered that students generally wanted to go on multiple ward call experiences. Between two doctors on a busy general surgery unit, it was difficult to provide this. We were impressed with how keen the students were to attend this type of experience.

This is a small study with only a few doctors and students involved. A larger study may reveal unanticipated problems. It is hoped that this pilot study will lead to a larger trial of the ward call buddy project that may identify these problems.

We plan to conduct a larger trial of this program, involving volunteer doctors who are not necessarily part of the study. This will help evaluate how robust our findings are. Furthermore, we aim to expand this study outside of general surgery, to other surgery departments and non-surgical departments (e.g. medicine).
Medical students have a limited understanding of what the responsibilities of junior doctors are during after hours ward call shifts. A buddy system can be beneficial in providing experience to medical students who are soon to embark on internship. More studies are required to fully elucidate the potential benefits of including ward call experience in the training of medical students.

\section{References}

1. Barzansky B and Etzel S. Medical schools in the United States, 2009-2010. Journal of the American Medical Association. 2010;304:1247-54.

2. Corriere MD, Hanson JL, Hemmer PA, and Denton GD. Overnight Call: A Survey of Medical Student Experiences, Attitudes, and Skills. Teaching and Learning in Medicine. 2013;25:64-70.

3. Goren WN, Leizman DS, La Rochelle J, Kogan JR. Overnight hospital experiences for medical students: results of the 2014 clerkship directors in internal medicine national survey. Journal of General Internal Medicine 2015;30:1-6.

\section{Declaration of interests}

AM: Nil to declare.

$\mathrm{AH}:$ Nil to declare.

\section{Acknowledgements}

We would like to thank the Department of General Surgery and the Acute Surgery Unit at the Royal Brisbane and Women's Hospital for allowing us to undertake this project in the Department. We would also like to thank the patients for allowing students to shadow the doctors in order to enhance their own learning and help future patients.

In particular, we would like to thank the Director of General Surgery Dr Barry O'Loughlin for allowing us to perform this study and for his encouragement. We would like to thank Professor Alan Gale, the Director of Clinical Training, for assisting with obtaining permission from the hospital, university, and relevant stakeholders and his enthusiasm. We would like to thank Amanda Felbermeyer, the student coordinator for the University of Queensland at the Royal Brisbane and Women's Hospital, for her assistance with facilitating the project.

\section{Ethical approval}

All relevant stakeholders with regards to this project were consulted prior to implementation. Full ethics approval was not deemed a necessity as students attending ward call shifts were not undertaking any additional responsibilities or impacting the hospital in any way that they would otherwise do in their normal, day-to-day clinical experience during normal office hours. Permission was obtained from the Hospital, Surgical Department, and University for undertaking this project.

\section{Conclusion}

